Pesq. Vet. Bras. 36(4):297-302, abril 2016 DOI: $10.1590 /$ S0100-736X2016000400008

\title{
Utilização de eletroquimioterapia para carcinoma de células escamosas tegumentar em felino ${ }^{1}$
}

\author{
Lucia M.G. Silveira ${ }^{2 *}$, Fernando M. Cunha², Carlos H.M. Brunner² e José G. Xavier ${ }^{2}$ \\ ABSTRACT.- Silveira L.M.G., Cunha F.M., Brunner C.H.M. \& Xavier J.G. 2016. [Employment \\ of electrochemotherapy for cutaneous squamous cell carcinoma in cats.] Utilização \\ de eletroquimioterapia para carcinoma de células escamosas tegumentar em felino. Pesqui- \\ sa Veterinária Brasileira 36(4):297-302. Setor de Cirurgia da Faculdade de Medicina Veteri- \\ nária, Universidade Paulista, Av. Tenente Julio Prado Neves 965, São Paulo, SP 02370-000, \\ Brazil. E-mail: Imgsilveira@gmail.com \\ Electrochemotherapy is characterized as a protocol which combines the use of antine- \\ oplastic agents and localized application of electric pulses (electroporation) to improve \\ the intracellular concentration of these agents, increasing its cytotoxic action. Bleomycin, \\ an antibiotic agent with antineoplastic properties, is a hydrophilic molecule, having a res- \\ tricted transport through the cellular membrane. However, when it is administered intra- \\ lesionally or intravenously and associated to electroporation, its cytotoxicity is maximized. \\ There were utilized 21 cats affected by cutaneous squamous cell carcinoma. The electro- \\ chemotherapy protocol was standardized using intravenous bleomycin sulfate at a dose of \\ $15 \mathrm{U} / \mathrm{m}^{2}$ body surface area. Electroporation was performed using an electrode composed of \\ needles and electric pulses with $1000 \mathrm{~V}$ voltage, in unipolar square wave and 100 microse- \\ conds duration, totalizing eight cycles. There was complete neoplastic remission in 21 cats \\ $(100 \%)$. There were no complications or side effects associated with the procedure. The \\ protocol studied in this work showed to be feasible, effective and safe for antineoplastic \\ therapy in feline cutaneous squamous cell carcinoma.
}

INDEX TERMS: Cat, neoplasia, electroporation, electrochemotherapy.

RESUMO.- A eletroquimioterapia compreende a utilização conjunta de fármacos antineoplásicos e aplicação regional de pulsos elétricos (eletroporação), maximizando a concentração intracelular destes fármacos, assim propiciando maior ação citotóxica. A bleomicina, fármaco antimicrobiano dotado de propriedade antineoplásica, apresenta restrita penetrabilidade na membrana celular, dada a sua hidrossolubilidade. Todavia, uma vez administrada via intralesional ou intravenosa associada à eletroporação, demonstra citotoxicidade potencializada. Foram utilizados 21 felinos acometidos por carcinoma de células escamosas tegumentar. Padronizou-se o protocolo eletroquimioterápico empregando-se sulfato de bleomicina, pela via intravenosa, na dose de $15 \mathrm{U} / \mathrm{m}^{2}$ de superfície corpórea. A eletroporação foi perfilada com eletrodo composto por agulhas,

\footnotetext{
${ }^{1}$ Recebido em 15 de agosto de 2015.

Aceito para publicação em 1 de março de 2016.

${ }^{2}$ Hospital Veterinário da Universidade Paulista (Unip), Av. Tenente Julio Prado Neves 965, São Paulo, SP 02370-000, Brasil. *Autor para correspondência: $\underline{\text { mgsilveira@gmail.com }}$
}

pulsos elétricos com tensão de $1000 \mathrm{~V}$, em onda quadrada unipolar, com duração de 100 microsegundos, totalizando oito ciclos. Verificou-se remissão neoplásica integral em 21 felinos inclusos no estudo (100\%). Inexistiram complicações e/ou efeitos adversos decorrentes do procedimento. 0 protocolo avaliado neste trabalho revelou-se exequível, eficaz e seguro na terapêutica antineoplásica de carcinoma de células escamosas tegumentar felino.

TERMOS DE INDEXAÇÃO: Gato, neoplasia, eletroporação, eletroquimioterapia.

\section{INTRODUÇÃO}

Estima-se que as neoplasias tegumentares representem alíquota próxima a 30\% do total de enfermidades oncológicas diagnosticadas no felino doméstico (Withrow \& Vail 2007). Dentre os cânceres cutâneos mais observados nessa espécie, sobressalta-se o carcinoma de células escamosas como neoplasia de grande incidência na rotina da clínica oncológica (Lucas \& Larsson 2006, Gross et al. 2007, Withrow \& Vail 2007). 
Apesar da provável etiopatogenia multifatorial do carcinoma de células escamosas, a radiação actínica e suas propriedades mutagênicas são fatores determinantes na manifestação deste tumor (Ferreira et al. 2006, Lucas \& Larsson 2006, Gross et al. 2007, Withrow \& Vail 2007). Junções mucocutâneas, incluindo plano e ponte nasal, regiões nasolabial e palpebral, assim como pavilhões auriculares e demais regiões tegumentares pobremente providos de pigmento melânico, pelagem curta e menos densa, são notoriamente propensos ao desenvolvimento de carcinoma de células escamosas, sobretudo naqueles animais continuadamente expostos à radiação solar (Ferreira et al. 2006, Lucas \& Larsson 2006, Gross et al. 2007, Withrow \& Vail 2007). Tal enfermidade caracteriza-se por massiva infiltração dérmica, por vezes estendida ao panículo adiposo subjacente (Gross et al. 2007). Metástases são incomuns, todavia há relatos de propagação neoplásica para linfonodos regionais e parênquima pulmonar (Gross et al. 2007).

A bibliografia oncológica veterinária disponibiliza inúmeros de protocolos para o tratamento do carcinoma de células escamosas felino, incluindo excisão cirúrgica, criocirurgia, terapia fotodinâmica, quimio e/ou radioterapia (Klein 2003, Ferreira et al. 2006, Lucas \& Larsson 2006, Buchholz et al. 2007, Hammond et al. 2007, Thomson 2007, Bexfield et al. 2008, Cunha et al. 2010). Entretanto, tais protocolos exibem limitações principalmente representadas por adversidades, resposta terapêutica e custo (Klein 2003, Ferreira et al. 2006, Lucas \& Larsson 2006, Buchholz et al. 2007, Hammond et al. 2007, Thomson 2007, Bexfield et al. 2008, Cunha et al. 2010).

A eletroporação consiste na aplicação regional de pulsos elétricos, curtos e de alta voltagem, os quais maximizam o potencial de transporte através da membrana celular, pela formação transitória de poros aquosos na bicamada lipídica, assim permitindo que macromoléculas sejam efetivamente conduzidas ao meio intracelular (Čemažar et al. 2001, Dujardin et al. 2002, Lebar et al. 2002, Pucihar et al. 2002, Gothelf et al. 2003, Chen et al. 2006, Serša et al. 2011, Gibot et al. 2013). A eficácia deste transporte encontra-se diretamente relacionada a um conjunto de fatores, principalmente representados por propriedades físicas da eletricidade (frequência de pulso, formato de onda e intensidade do campo elétrico) e características fisicoquímicas dos fármacos mais utilizados no protocolo (Dujardin et al. 2002, Lebar et al. 2002, Pucihar et al. 2002, Gothelf et al. 2003, Chen et al. 2006, Serša et al. 2011, Gibot et al. 2013). A eletroquimioterapia constitui um procedimento terapêutico que agrega o emprego de fármacos antineoplásicos à eletroporação, visando à maximização da concentração intracelular destes fármacos, então propiciando maior ação citotóxica dos mesmos (Čemažar et al. 2001, Gothelf et al. 2003, Serša et al. 2006, 2011, Larkin et al. 2007, Meschini et al. 2012, Gibot et al. 2013). Muitos quimioterápicos são estruturalmente constituídos por moléculas hidrófilas, exibindo assim restrição no transporte através da membrana celular. Contudo, uma vez simultaneamente administrados à eletroporação, exibem extrema potencialização do efeito terapêutico, em diminutas dosagens (Čemažar et al. 2001, Gothelf et al. 2003, Aminkov \& Manov 2004, Giardino et al.
2006, Serša et al. 2006, 2011, Larkin et al. 2007, Meschini et al. 2012, Gibot et al. 2013). Dentre os mencionados fármacos, enfoque foi dado à amplificação da citotoxicidade exercida pela bleomicina, antibiótico com propriedade antitumoral, a qual, uma vez combinada à aplicação regional de pulsos elétricos, demonstra otimização de seus efeitos terapêuticos antitumorais (Čemažar et al. 2001, Gothelf et al. 2003, Serša et al. 2006, 2011, Gibot et al. 2013).

Ampla bibliografia referente à utilização de eletroquimioterapia na clínica oncológica humana e veterinária encontra-se disponível (Čemažar et al. 2001, Gothelf et al. 2003, Aminkov \& Manov 2004, Giardino et al. 2006, Serša et al. 2006, 2011, Spugnini et al. 2009, Silveira et al. 2010, 2011). Os resultados até então obtidos, mostram-se promissores, sobretudo representados por remissão neoplásica parcial ou integral, efeitos adversos irrelevantes e diminuto percentual ou aumento no intervalo de tempo livre de recidiva e/ou metástase (Čemažar et al. 2001, Gothelf et al. 2003, Aminkov \& Manov 2004, Giardino et al. 2006, Serša et al. 2006, 2011, Spugnini et al. 2009, Silveira et al. 2010, 2011).

Este estudo objetivou avaliar a exequibilidade, efetividade e segurança da eletroquimioterapia em carcinoma de células escamosas tegumentar felino, no intuito da contribuição relativa à otimização de sua terapia, com consequente melhora no prognóstico para os pacientes acometidos pela doença.

\section{MATERIAL E MÉTODOS}

Foram utilizados neste estudo 21 felinos acometidos por formação neoplásica tegumentar solitária, atendidos no Serviço de Cirurgia do Hospital Veterinário da Faculdade de Medicina Veterinária da Universidade Paulista, São Paulo, Capital, no período de abril de 2012 a abril de 2013. 0 estudo foi conduzido conforme diretrizes referentes à experimentação animal, pré-determinadas pelo Conselho de Ética da Universidade Paulista (Protocolo no. 009/11, aprovado e registrado em ata de reunião do Conselho de Ética em 17 de março de 2011). Os proprietários desses pacientes foram previamente orientados sobre os procedimentos relativos ao protocolo.

Quadro 1. Estadiamento clínico para carcinoma de células escamosas felino segundo o sistema TNM (Withrow \& Vail 2007)

\begin{tabular}{cl}
\hline Tumor primário & \\
Tis & Carcinoma in situ \\
T0: & Sem evidências de tumor \\
T1: & Tumor < $2 \mathrm{~cm}$ de diâmetro, superficial \\
T2: & Tumor entre 2 - 5 cm de diâmetro com invasão mínima \\
T3: & Tumor > $5 \mathrm{~cm}$ de diâmetro com invasão para \\
& subcutâneo \\
T4: & Tumor invadindo outras estruturas como fáscia, \\
& osso ou cartilagem \\
Linfonodo (N) & \\
N0: & Sem evidências de envolvimento de linfonodo regional \\
N1: & Linfonodo ipsilateral móvel \\
& N1a: sem aumento de volume \\
N2: & N1b: com aumento de volume \\
& Linfonodo contralateral ou bilateral móveis \\
& N2a: sem aumento de volume \\
N3: & N2b: com aumento de volume \\
Metástase (M) & Linfonodos fixos \\
M0: & Sem evidências de metástase distante \\
M1: & Metástase distante detectada
\end{tabular}


Todos os felinos passaram por avaliação preliminar de minucioso exame físico e pelos complementares pertinentes, assim representados por hematológico, perfil bioquímico, radiográfico torácico e ultrassonográfico abdominal, investigando-se enfermidades coexistentes e/ou neoplasia intracavitária primária ou metastática. Submeteu-se cada lesão à análise micromorfológica, após colheita de espécimes via técnica incisional e fixação dos mesmos em solução tamponada de formalina a 10\%. A histopatologia objetivou o delineamento do tipo neoplásico, origem tecidual e seu comportamento biológico. Todos os pacientes foram submetidos a anestesia geral durante a realização de biópsia incisional e do protocolo eletroquimioterápico.

0 estadiamento clínico dos carcinomas de células escamosas tegumentares abordados neste estudo foi realizado em concordância com o sistema TNM (tumor/linfonodo/metástase) (Withrow \& Vail 2007) (Quadro 1).

Perfilou-se a eletroquimioterapia utilizando-se sulfato de bleomicina, via intravenosa, na dose de $15 \mathrm{U} / \mathrm{m}^{2}$ de superfície corpórea. Cinco minutos após a aplicação deste fármaco, administraram-se pulsos elétricos (eletroporação) sobre toda a extensão da neoformação por intermédio de aparelhagem específica. A eletroporação foi realizada com eletrodo composto por oito agulhas de aço inoxidável, paralelamente dispostas e equidistantes $(0,7 \mathrm{~cm})$. Pulsos elétricos com tensão de $1000 \mathrm{~V}$ em onda quadrada unipolar, com duração de 100 microsegundos, totalizando oito ciclos, foram aplicados em todo volume tumoral . Todo procedimento foi mensalmente repetido até a remissão neoplásica macroscópica integral.
Os animais foram reavaliados 15, 30 dias e 60 dias após a realização do protocolo. Constatada a regressão tumoral macroscópica total, todos os pacientes foram monitorados através de retornos bimestrais durante 12 meses.

\section{RESULTADOS}

A análise histológica das lesões abordadas neste estudo revelou 21 casos de carcinoma de células escamosas tegumentar. Destes, sete situavam-se em pavilhão auricular, cinco em pálpebras e nove em plano nasal (Fig.1A, 2A, 3A). Não havia evidência física, laboratorial e/ou por imagem de metástase nestes pacientes. As informações referentes à resenha dos animais, localização anatômica e estadiamento clínico de todas as neoformações encontram-se pormenorizadas no Quadro 2. Inexistiram adversidades decorrentes da administração intravenosa de sulfato de bleomicina e/ ou da eletroporação. Não se evidenciaram lesões térmicas e/ou hemorrágicas imediatas advindas do contato dos eletrodos com o tecido submetido ao protocolo.

Aos 15 dias após aplicação constatou-se remissão parcial das neoformações, assim como regiões erodidas e cobertas por crostas. A reavaliação clínica realizada 30 dias após o protocolo, apontou remissão tumoral total em 12 pacientes submetidos a apenas uma sessão do protocolo $(57,1 \%)$ (Fig.1B, 2B, 3B). Neoplasias que exibiram resposta parcial após uma sessão do protocolo $(n=9)(42,9 \%)$ foram

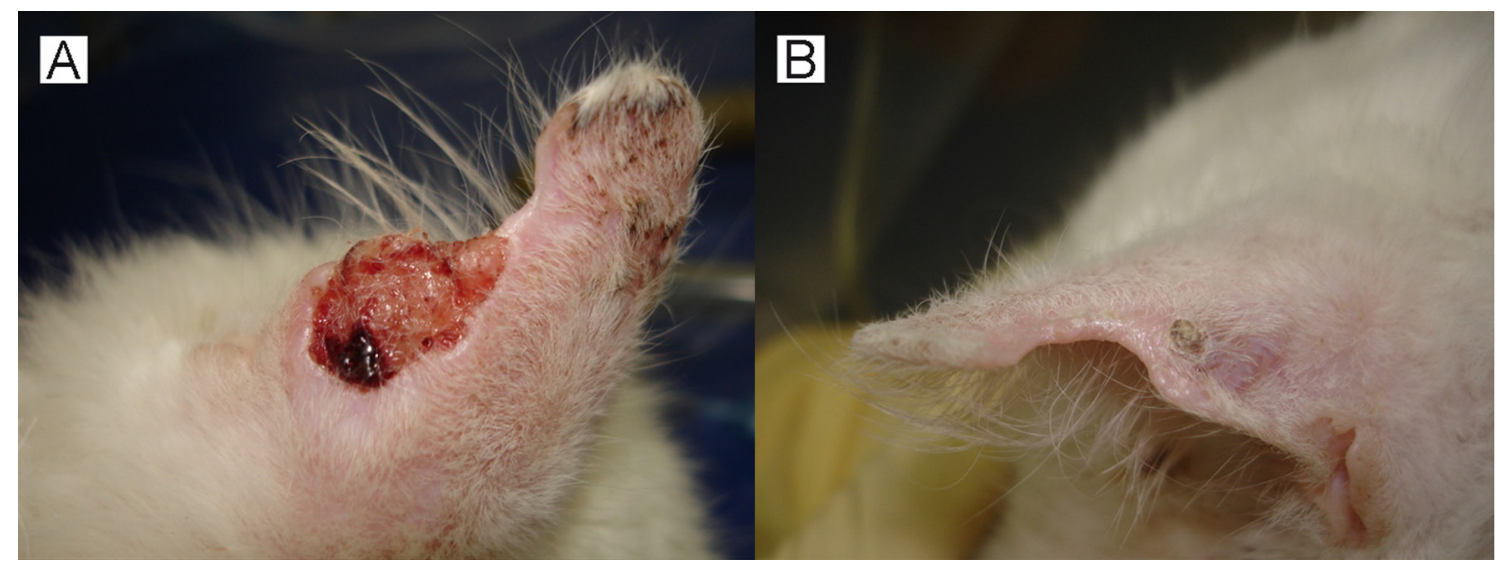

Fig.1. (A) Aspecto macroscópico de carcinoma de células escamosas tegumentar felino em região auricular. (B) Remissão neoplásica integral 30 dias após a primeira sessão de eletroquimioterapia.

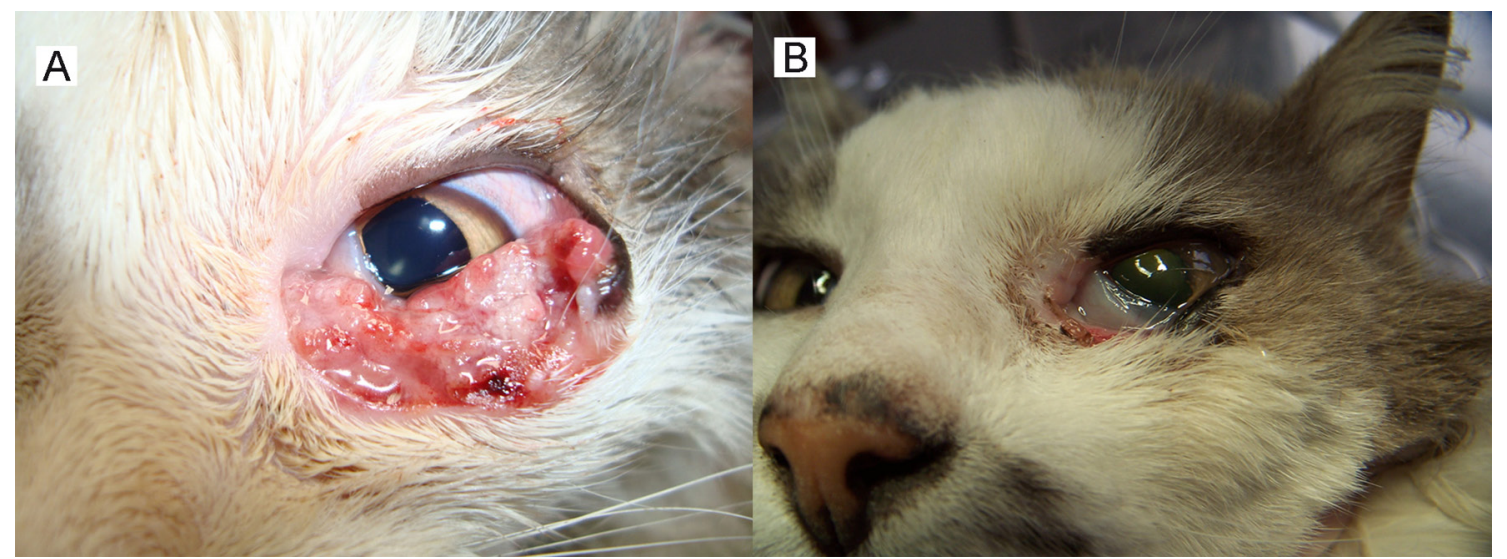

Fig.2. (A) Aspecto macroscópico de carcinoma de células escamosas tegumentar felino em região palpebral. (B) Remissão neoplásica integral após duas sessões eletroquimioterápicas (60 dias). 


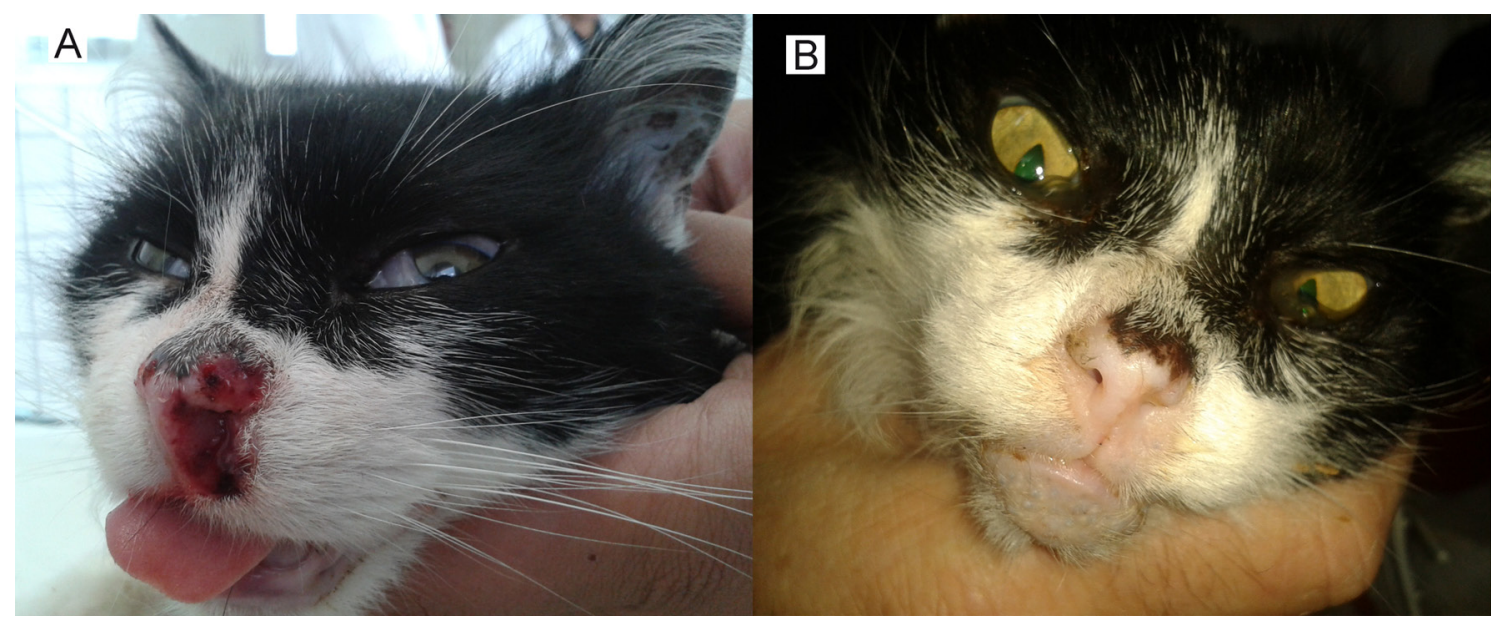

Fig.3. (A) Aspecto macroscópico de carcinoma de células escamosas tegumentar felino em região nasal. (B) Remissão neoplásica integral após duas sessões de eletroquimioterapia (60 dias).

Quadro 2. Caracterização dos animais, localização anatômica e estadiamento tumoral dos gatos acometidos por carcinoma espinocelular tegumentar. FMV/UNIP, São Paulo, 2012

\begin{tabular}{cccccc}
\hline Felino & Raça & Sexo & Idade (anos) & $\begin{array}{c}\text { Localização } \\
\text { anatômica }\end{array}$ & $\begin{array}{c}\text { Estadiamento } \\
\text { clínico }\end{array}$ \\
\hline 1 & SRD & F & 8 & pavilhão auricular & T2 N0 M0 \\
2 & SRD & M & 16 & pavilhão auricular & T2 N0 M0 \\
3 & SRD & F & 15 & pavilhão auricular & T2 N0 M0 \\
4 & SRD & M & 11 & pavilhão auricular & T2 N0 M0 \\
5 & SRD & M & 11 & pavilhão auricular & T2 N0 M0 \\
6 & SRD & F & 12 & pavilhão auricular & T1 N0 M0 \\
7 & SRD & F & 14 & pavilhão auricular & T1 N0 M0 \\
8 & SRD & F & 10 & plano nasal & T1 N0 M0 \\
9 & SRD & M & 10 & plano nasal & T1 N0 M0 \\
10 & SRD & F & 15 & plano nasal & T1 N0 M0 \\
11 & SRD & M & 5 & plano nasal & T1 N0 M0 \\
12 & SRD & M & 5 & plano nasal & T1 N0 M0 \\
13 & SRD & F & 16 & plano nasal & T1 N0 M0 \\
14 & SRD & F & 8 & plano nasal & T1 N0 M0 \\
15 & SRD & F & 15 & plano nasal & T1 N0 M0 \\
16 & SRD & F & 12 & plano nasal & T1 N0 M0 \\
17 & SRD & M & 8 & pálpebra & T2 N0 M0 \\
18 & SRD & F & 4 & pálpebra & T2 N0 M0 \\
19 & SRD & M & 15 & pálpebra & T2 N0 M0 \\
20 & SRD & M & 12 & pálpebra & T2 N0 M0 \\
21 & SRD & F & 13 & pálpebra & T1 N0 M0
\end{tabular}

a Sem raça definida.

novamente submetidas ao protocolo, alcançando-se, então, regressão macroscópica completa dos tumores. Vinte e uma neoformações submetidas ao protocolo (100\%) demonstraram regressão total.

Todos os pacientes foram reavaliados em retornos bimestrais através de minucioso exame físico e subsidiários pertinentes durante doze meses, não demonstrando, neste período, sinais clínicos e/ou alterações laboratoriais e/ou por imagem de recidiva neoplásica e/ou metástase.

\section{DISCUSSÃO}

Diversos protocolos destinam-se à terapêutica de carcinoma de células escamosas tegumentar felino. Tais procedimentos demonstram expressivas disparidades relativas a eficácia, duração do tratamento, período de recuperação do paciente, segurança e onerosidade (Klein 2003, Ferreira et al. 2006, Lucas \& Larsson 2006, Buchholz et al. 2007, Hammond et al. 2007, Thomson 2007, Bexfield et al. 2008, Cunha et al. 2010). Assim, estudos suplementares estão sendo amplamente desenvolvidos na busca por alternativas terapêuticas mais efetivas e seguras (Čemažar et al. 2001, Gothelf et al. 2003, Aminkov \& Manov 2004, Chen et al. 2006, Withrow \& Vail 2007, Serša et al. 2011, Meschini et al. 2012, Gibot et al. 2013). A bibliografia oncológica humana e veterinária qualificou a eletroquimioterapia como método exequível, eficaz e seguro (Čemažar et al. 2001, Gothelf et al. 2003, Aminkov \& Manov 2004, Chen et al. 2006, Giardino et al. 2006, Larkin et al. 2007, Spugnini et al. 2009, Silveira et al. 2010, 2011). Constitui protocolo indicado no tratamento de inúmeros tumores, excetuando-se os de origem óssea e hematopoiética (Čemažar et al. 2001, Aminkov \& Manov 2004, Giardino et al. 2006, Serša et al. 2006). Este estudo obteve resultados similares e igualmente relevantes às descrições anteriores que demonstraram, quando adequadamente conduzidos, resultados bastante promissores, com enfoque naqueles obtidos na terapêutica de carcinoma de células escamosas tegumentar felino (Čemažar et al. 2001, Spugnini et al. 2009).

A administração intravenosa de sulfato de bleomicina revelou-se viável, eficaz e segura. Inexistiram adversidades atribuíveis ao uso deste fármaco pela via mencionada, dado este também concordante com os descritos em parte da literatura compilada (Čemažar et al. 2001, Gothelf et al. 2003, Aminkov \& Manov 2004).

Lesões tegumentares térmicas advindas do contato dos eletrodos com o tecido onde foi aplicado a eletroporação são passíveis de ocorrência. Tal complicação não foi verificada nos pacientes da amostragem adotada neste estudo e foi apenas eventualmente constatada em trabalhos prévios (Čemažar et al. 2001, Dujardin et al. 2002, Lebar et al. 2002, Pucihar et al. 2002, Gothelf et al. 2003, Chen et al. 2006, Serša et al. 2006, Silveira et al. 2010, 2011).

A(s) molécula(s), a classe farmacológica, a dosagem e a via de administração dos agentes antineoplásicos, assim como as características dos eletrodos e intensidade, núme- 
ro e tipo de ondas produzidas pelos pulsos elétricos, variaram na literatura consultada (Čemažar et al. 2001, Dujardin et al. 2002, Lebar et al. 2002, Pucihar et al. 2002, Gothelf et al. 2003, Aminkov \& Manov 2004, Chen et al. 2006, Giardino et al. 2006, Serša et al. 2006, 2011, Larkin et al. 2007, Spugnini et al. 2009, Silveira et al. 2010, Gibot et al. 2013). Todavia, a eletroquimioterapia realizada com sulfato de bleomicina na dose de $15 \mathrm{U} / \mathrm{m}^{2}$ de superfície corpórea, via intravenosa em associação à administração regional de pulsos elétricos com intensidade de $1000 \mathrm{~V}$ por $100 \mathrm{mi}$ crosegundos, em onda quadrada unipolar, totalizando oito pulsos, demonstrou-se amplamente eficaz na remissão de carcinoma de células escamosas tegumentar submetido ao protocolo neste estudo.

O número de sessões eletroquimioterápicas variou de uma a duas até constatação de regressão tumoral completa ao exame físico. Segundo dados reunidos, o número de sessões a realizar encontra-se diretamente relacionado ao volume, ao tipo tumoral e ao comportamento biológico da neoplasia abordada (Gothelf et al. 2003, Serša et al. 2006, Silveira et al. 2010, 2011, Gibot et al. 2013).

O monitoramento clínico após a remissão tumoral total compreendeu o período de doze meses, no qual não houve indícios clínicos, laboratoriais e/ou por imagem de recidiva ou propagação tumoral. 0 potencial metastático encontra-se diretamente atrelado a fatores como origem e natureza da neoplasia considerada e seu estadiamento clínico, terapia específica utilizada e salubridade imunológica do paciente. Mecanismos ainda não completamente elucidados indicam que a eletroquimioterapia promove estímulo imunológico celular e humoral, otimizando a inibição de recidivas e/ou metástases (Gothelf et al. 2003, Serša et al. 2006, Silveira et al. 2010).

\section{CONCLUSÕES}

A eletroquimioterapia demonstrou ser aplicável, eficaz e segura na terapêutica de carcinoma de células escamosas tegumentar felino.

Dentre as vantagens intrínsecas ao procedimento, destacaram-se ausência de toxicidade atribuível à administração intravenosa de sulfato de bleomicina, rapidez e praticidade na execução do protocolo eletroquimioterápico, inexistência de complicações trans/pós-terapêuticas e baixa onerosidade.

Cabe ainda ressaltar a manutenção da integridade anatomofisiológica da pele no local acometido e que não foram observadas recidivas e/ou metástases nos pacientes submetidos à eletroquimioterapia e submetidos a reavaliações clínicas bimestrais por 12 meses.

\section{REFERÊNCIAS}

Aminkov B. \& Manov V. 2004. Electrochemotherapy: a novel method of treatment of malignant tumours in the dog. Bulgarian J. Vet. Med. 7(4): 209-213.

Bexfield N.H., Stell A.J., Gear R.N. \& Dobson J.M. 2008. Photodynamic therapy of superficial nasal planum squamous cell carcinomas in cats: 55 cases. J. Vet. Intern. Med. 22(6):1385-1389.

Buchholz J., Walt H., Gräfe S., Bley C.R. \& Kaiser-Hotz B. 2007. Photodynamic therapy of feline cutaneous squamous cell carcinoma using a newly developed liposomal photosensitizer: preliminary results concerning drugs safety and efficacy. J. Vet. Intern. Med. 21:4:770-775.

Chen C., Smye S.W., Robinson M.P. \& Evans J.A. 2006. Membrane electroporation theories: a review. Med. Biol. Engineering and Computing 44(1/2):5-14.

Čemažar M., Miklavčic D., Mir L.M., Belehradek J Jr, Bonnay M., Fourcault D. \& Serša G. 2001. Electrochemotherapy of tumours resistant to cisplatin: a study in a murine tumour model. Eur. J. Cancer 37(9):1166-1172.

Cunha S.C.S., Carvalho L.A.V., Canary P.C., Reisner M., Corgozinho K.B., Souza H.J.M. \& Ferreira A.M.R. 2010. Radiation therapy for feline cutaneous squamous cell carcinoma using a hypofractionated protocol. J. Feline Med. Surg. 12(4):306-313.

Dujardin N., Staes E., Kalia Y., Clarys P., Guy R. \& Prèat V. 2002. In vivo assessment of skin electroporation using square wave pulses. J. Controlled Release 79(1/3):219-227.

Ferreira I., Rahal S.C., Ferreira J. \& Corrêa T.P. 2006. Terapêutica no carcinoma de células escamosas cutâneo em gatos. Ciência Rural 36(3):10271033.

Giardino R., Fini M., Bonazzi V., Cadossi R., Nicolini A. \& Carpi A. 2006. Electrochemotherapy: a novel approach to the treatment of metastatic nodules on the skin and subcutaneous tissues. Biomed. Pharmacother. 60(8):458-462.

Gothelf A., Mir L.M. \& Gehl J. 2003. Electrochemotherapy: results of cancer treatment using enhanced delivery of bleomycin by electroporation. Cancer Treatment Reviews 29(5):371-387.

Gibot L., Wasungu L., Teissié J. \& Rols M.P. 2013. Antitumor drug delivery in multicellular spheroids by electropermeabilization. J. Controlled Release 167(2):138-147.

Gross T.L., Ihrke P.J., Walder E.J. \& Affolter V.K. 2007. Neoplasias epiteliais e outros tumores, p.546-81. In: Ibid. (Eds), Doenças de Pele do Cão e do Gato: diagnóstico clínico e histopatológico. 2ª ed., Roca, São Paulo.

Hammond G.M., Gordon I.K., Theon A.P. \& Kent M.S. 2007. Evaluation of strontium $\mathrm{Sr} 90$ for the treatment of superficial squamous cell carcinoma of the nasal planum in cats: 49 cases (1990-2006). J. Am. Vet. Med. Assoc. 231(5):736-741.

Klein M. 2003. Multimodality therapy for head and neck cancer. Vet. Clin. North Am., Small Anim. Pract. 33(3):615-628.

Larkin J.O., Collins C.G., Aarons S., Tangney M., Whelan M., O’Reily S., Breathnach O., Soden D.M. \& O'Sullivan G.C. 2007. Electrochemotherapy: aspects of preclinical development and early clinical experience. Annals of Surgery 245(3):469-479.

Lebar A.M., Serša G., Kranjc S., Grošelj A. \& Miklavčič D. 2002. Optimization of pulse parameters in vitro for in vivo electrochemotherapy. Anticancer Research 22(3):1731-1736.

Lucas R. \& Larsson C.E. 2006. Crioterapia na clínica veterinária: avaliação da praticabilidade, e efetividade em carcinoma espinocelular de felinos. Braz. J. Vet. Res. Anim. Sci. 43(Supl.):33-42.

Meschini S., Condello M., Lista P., Vicenzi B., Baldi A., Citro G., Arancia G. \& Spugnini E.P. 2012. Electroporation adopting trains of biphasic pulses enhances in vitro and in vivo the cytotoxic effect of doxorubicin on multidrug resistant colon adenocarcinoma cells (LoVo). Eur. J. Cancer 48(14):2236-2243.

Pucihar G., Mir L.M. \& Miklavcic D. 2002. The effect of pulse repetition frequency on the uptake into electropermeabilized cells in vitro with possible applications in electrochemotherapy. Bioelectrochemistry 57(2): 167-172.

Serša G., Čemazar M., Miklavčič D. \& Rudolf Z. 2006. Electrochemotherapy of tumours. Radiol. Oncol. 40(3):163-174.

Serša G., Cufer T., Paulin S.M., Cemazar M. \& Snoj M. 2011. Electrochemotherapy of chest wall breast cancer recurrence. Cancer Treatment Reviews 38(5):379-86.

Silveira L.M.G., Brunner C.H.M., Cunha F.M., Futema F., Calderaro F.F. \& Kozlowski D. 2010. Utilização de eletroquimioterapia em neoplasias de origem epitelial e mesenquimal localizadas em pele ou mucosas de cães. Braz. J. Vet. Res. Anim. Sci. 47(1):55-66.

Silveira L.M.G., Brunner C.H.M., Cunha F.M., Rocha M. \& Futema F. 2011. 
Eletroquimioterapia em adenocarcinoma perianal canino. J. Health Sci. Inst. 29(2):136-138.

Spugnini E.P., Vincenzi B., Citro G., Tonini G., Dotsinsky I., Mudrov N. \& Baldi A. 2009. Electrochemotherapy for the treatment of squamous cell carcinoma in cats: a preliminary report. Vet. J. 179(1):117-120.
Thomson M. 2007. Squamous cell carcinoma of the nasal planum in cats and dogs. Clin. Tech. Small Anim. Pract. 22(2):42-45.

Withrow S.J. \& Vail D.M. 2007. Tumors of skin and subcutaneous tissues, p.375-401. In: Ibid. (Eds), Withrow and MacEwen's Small Animal Clinical Oncology. Saunders Elsevier, St Louis. 